\title{
The specificity of reciprocity: Young children reciprocate more generously to those who intentionally benefit them
}

\author{
Amrisha Vaish ${ }^{*}$, Robert Hepach ${ }^{1}$, Michael Tomasello ${ }^{2}$ \\ Max Planck Institute for Evolutionary Anthropology, 04103 Leipzig, Germany
}

\section{A R T I C L E I N F O}

\section{Article history:}

Received 29 June 2017

Revised 26 October 2017

Available online 8 December 2017

\section{Keywords:}

Reciprocity

Sharing

Cooperation

Prosocial behavior

Gratitude

Prosocial development

\begin{abstract}
A B S T R A C T
Young children engage in direct reciprocity, but the mechanisms underlying such reciprocity remain unclear. In particular, prior work leaves unclear whether children's reciprocity is simply a response to receiving benefits (regardless of whether the benefits were intended) or driven by a mechanism of rewarding or preferring all benefactors (regardless of whom they benefited). Alternatively, perhaps children engage in genuine reciprocity such that they are particularly prosocial toward benefactors who intentionally provided them with benefits. Our findings support this third, richer possibility; the 3-year-olds who received benefits through the good intentions of a benefactor were subsequently more generous toward the benefactor than children who either (a) received the same benefits from the benefactor unintentionally or (b) observed the benefactor bestow the same benefits on another individual. Thus, young children are especially motivated to benefit those who have demonstrated goodwill toward them, suggesting, as one possible mechanism, an early sense of gratitude.
\end{abstract} (c) 2017 Elsevier Inc. All rights reserved.

\footnotetext{
* Corresponding author at: Department of Psychology, University of Virginia, Charlottesville, VA 22904, USA.

E-mail address: vaish@virginia.edu (A. Vaish).

${ }^{1}$ Current address: Department of Research Methods in Early Child Development, Faculty of Education, Leipzig University, 04109 Leipzig, Germany.

${ }^{2}$ Current address: Department of Psychology \& Neuroscience, Duke University, Durham, NC 27708, USA.
} 


\section{Introduction}

Humans are extraordinarily prosocial; we help and share with others, comfort those in distress, and even pay costs to ensure the well-being of others (Tomasello, 2009). Strikingly, these prosocial behaviors are often directed at genetically unrelated individuals. This poses a puzzle: Why do humans provide benefits to others when neither they nor their genetic relations gain from those benefits?

One principal explanation is direct reciprocity wherein favors are exchanged over repeated interactions between the same two individuals (Nowak, 2006; Trivers, 1971). By taking turns paying costs and receiving benefits, both individuals benefit in the long term. Indeed, extensive work demonstrates that adults readily engage in direct reciprocity and that direct reciprocity is an important way in which human cooperation is maintained (e.g., Fehr \& Fischbacher, 2004).

Developmental research shows that even young children engage in direct reciprocity. For instance, 3-year-olds share more resources with a partner if that partner had previously shared resources with them than if the partner had not previously shared with them (Warneken \& Tomasello, 2013; see also Levitt, Weber, Clark, \& McDonnell, 1985). By preschool age, children also expect reciprocity from those they had previously benefited (Paulus, 2016; Sebastián-Enesco \& Warneken, 2015). Thus, direct reciprocity is a fundamental part of human cooperation from early in ontogeny.

What remains unclear, however, is why children show reciprocal behavior. One possibility is that children evaluate all generous individuals positively and, thus, act prosocially toward them even if the individuals were generous toward third parties. In line with this, prior work has shown that toddlers and preschoolers prefer and are more prosocial toward individuals who are prosocial rather than antisocial toward third parties (e.g., Dahl, Schuck, \& Campos, 2013; Hamlin, Wynn, Bloom, \& Mahajan, 2011; Kenward \& Dahl, 2011; Vaish, Carpenter, \& Tomasello, 2010). Thus, it may be that what is interpreted as direct reciprocity in young children is in fact driven by a more general mechanism of rewarding or preferring prosocial individuals.

A second possibility is that children feel happy whenever they receive benefits regardless of whether or not the giver intended to benefit them, and this motivates them to act prosocially. Indeed, a positive mood does promote adults' prosocial behavior (see Carlson, Charlin, \& Miller, 1988), and this may reasonably be true in young children as well (see, e.g., Aknin, Hamlin, \& Dunn, 2012). Note that in prior work, 21-month-olds did respond to their benefactors' intentions and were more likely to help an adult who previously intended but failed to benefit them than an adult who unwillingly benefited them (Dunfield \& Kuhlmeier, 2010). However, young children may judge all well-intentioned benefactors more positively than ill-intentioned ones even if the good intentions are directed toward third parties. In line with this, 3-year-olds evaluate positively and are more prosocial toward individuals who have helpful rather than harmful intentions toward third parties (Killen, Mulvey, Richardson, Jampol, \& Woodward, 2011; Nobes, Panagiotaki, \& Pawson, 2009; Vaish et al., 2010).

A third, richer possibility is that young children take both of the above factors into account (whether the children received benefits and whether the benefactor intended to benefit the children), such that they are particularly appreciative and prosocial toward benefactors who intended to benefit them. This specificity in children's reciprocity would indicate that children are particularly motivated to invest in the well-being of those individuals who mean them well, thereby engaging in and helping to sustain cooperation from early in ontogeny.

Strikingly, developmental research has not teased apart these possibilities, leaving unclear what mechanisms underlie young children's emerging reciprocity. Our aim was to fill this gap in our knowledge. Toward this end, we adapted the contingent reciprocity paradigm used by Warneken and Tomasello (2013). In that study, when 2- and 3-year-olds ran out of items that they needed to play a game, a puppet either shared or did not share its game items with them. Subsequently, the puppet ran out of game items and children could share some of their game items with the puppet. We modified and extended this basic paradigm in order to disentangle the mechanisms described above. Specifically, in the current study, a puppet provided game items either to the child or to another recipient and did so either with the intention of sharing or not. The child then had the opportunity to share game items with the benefactor. 
Warneken and Tomasello (2013) found that 3-year-old children were more likely to share with the puppet if the puppet had previously shared with them, whereas 2-year-olds did not show such reciprocal sharing. Based on this finding and the finding that 3-year-olds follow principles of reciprocity in hypothetical sharing situations (Olson \& Spelke, 2008), and because we were interested in delving further into the mechanisms underlying reciprocal sharing, in the current study we focused on 3-yearolds. We predicted that if 3-year-olds engage in genuine reciprocity toward their benefactor, then they should be more generous when the benefactor intentionally provided them with benefits than in all other conditions.

To further explore the phenomenon, we were interested in children's prosocial behavior and social decisions outside the immediate context within which they received benefits. It is possible that reciprocity extends beyond the immediate context and continues to influence the beneficiary's behavior even in novel situations where the beneficiary has the opportunity to benefit the benefactor or to select the benefactor as a social partner. On the other hand, and in line with classic conceptions of direct reciprocity as giving and taking in turns (e.g., Axelrod \& Hamilton, 1981; Fehr \& Fischbacher, 2004; Trivers, 1971), it is possible that once beneficiaries have reciprocated, they might not continue to bestow favors (assuming that the benefactor provides no additional benefits). To explore these ideas, we conducted three additional secondary tasks after the primary Sharing task. Two tasks assessed children's prosocial behavior (helping and resource distribution) toward the giver, and a third task assessed children's preference for the giver as a social partner. Given their exploratory nature, we did not have specific predictions about these secondary tasks.

\section{Method}

\section{Participants}

A total of 85 3-year-old children were tested, of which 5 were excluded due to equipment failure $(n=4)$ or unwillingness to participate $(n=1)$. Thus, the final sample consisted of 803 -year-olds (40 girls; $M_{\mathrm{age}}=3$ years 6 months, $S D=1$ month 7 days, range $=3$ years 3 months 24 days to 3 years 7 months 30 days). Children were recruited from and tested at their day-care centers in a mediumsized German city. No ethnicity or socioeconomic status data were collected, but approximately $98 \%$ of the population from which the sample was drawn is native German and the population includes a broad mix of socioeconomic backgrounds. All children's parents had given permission for the children to participate in child development studies. On average, about $60 \%$ of parents at any particular day-care center give their consent, with a range of $20-100 \%$.

\section{Materials}

Two large hand puppets ( $\sim 40 \mathrm{~cm}$ high), whose mouths and hands could be controlled by puppeteers, were used. Both puppets wore gender-neutral clothes and were given names matching the gender of the child. For the Sharing tasks, two jingle boxes and two marble runs were used. Players (the child and the two puppets) needed specific items to play the games: wooden cubes for the jingle boxes and marbles for the marble runs (adapted from Warneken \& Tomasello, 2013). Both games involved placing the items into apparatuses, where the items disappeared (see Figs. 1 and 2). Specifically, the jingle box consisted of a large cardboard box with a tube on one side as an opening. When a player threw a small wooden cube into the tube, it created a jingle sound due to a xylophone installed inside and then landed in the box. The marble run consisted of two sloping tracks that ended in a cardboard box. The beginning of the marble run was designed such that only one marble could be placed there at a time (so that the child needed to roll down his or her marbles one at a time rather than all together). A marble placed at the top rolled down the tracks and ended in the box.

In addition, there were three colored mats (red, blue, and green) on which the child and the two puppets sat as well as containers (also red, blue, and green) to hold the wooden cubes or marbles for each player. The materials for the exploratory tasks are described below. 


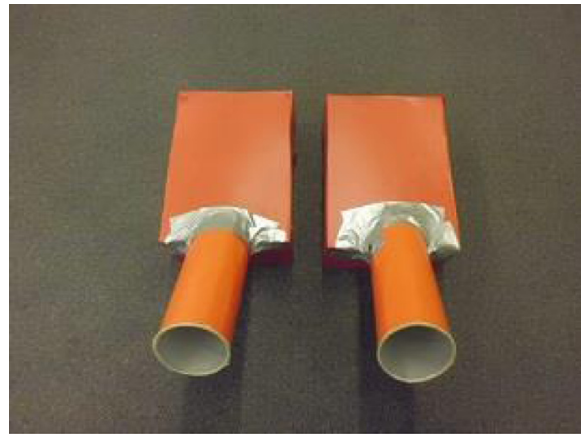

A

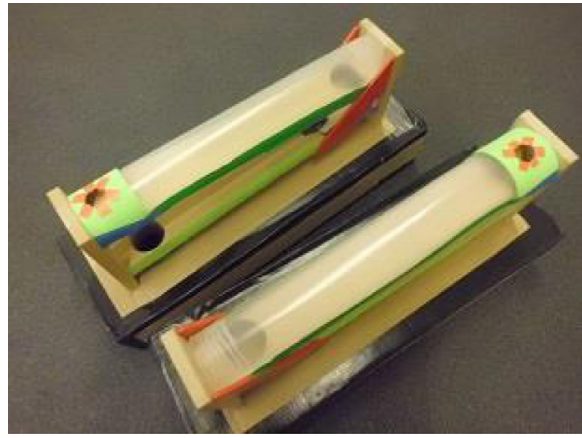

B

Fig. 1. The two jingle boxes (A) and two marble runs (B) used for the Sharing tasks.

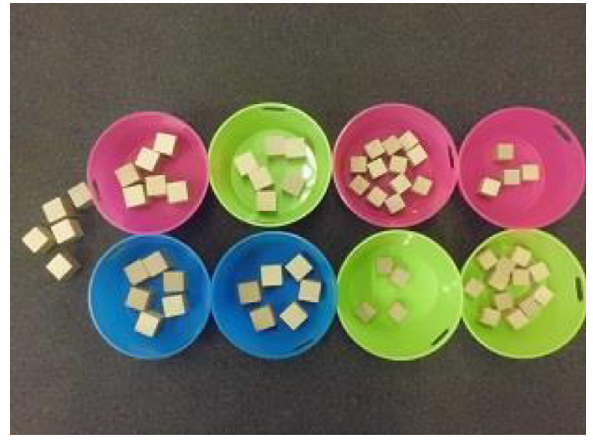

A

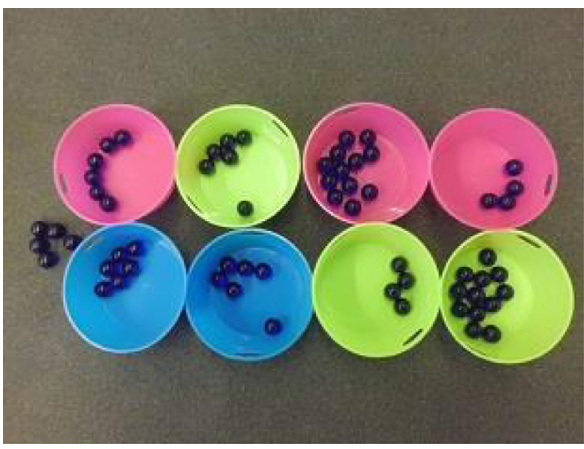

B

Fig. 2. The cubes used with the jingle boxes (A) and the marbles used with the marble runs (B). Both panels show the items as they were set up for the Shared-with-child condition; the five loose items on the left were used during the demonstration phase, and each pair of containers was used for each round of the game (i.e., leftmost pair used on the first round, second pair from the left used on the second round, etc.). The colors of the containers determined which two of the three players played on each round. (For interpretation of the references to color in this figure legend, the reader is referred to the web version of this article.)

\section{Procedure}

The study had a 2 (Beneficiary: child or receiver) $\times 2$ (Action: shared or fell) between-participants factorial design. Children were randomly assigned to one of four conditions: Shared-with-child, Fellnear-child, Shared-with-receiver, or Fell-near-receiver.

Children first warmed up with an experimenter (the moderator) in their playroom for 3-5 min. The moderator then brought each child to the testing room and introduced the child to two puppets (each played by a different experimenter). Throughout the procedure, the moderator treated the puppets and the child very similarly such as by instructing all of them on the games. This was intended to make the child see the puppets as being of equal status and, thus, to feel comfortable in interacting with them (see Warneken \& Tomasello, 2013). The same female experimenter always played the role of moderator across all children. The puppeteers were two female experimenters who switched roles between giver puppet and receiver puppet across children. Because one puppeteer was unavailable after 64 children had been tested, a new female experimenter replaced her for the remaining 16 children (4 per condition). All experimenters were blind to hypotheses. 
All children first took part in the Sharing tasks (which consisted of two games, both in the same condition), followed by the three exploratory tasks. We describe each in turn.

\section{Sharing tasks}

Each puppet was seated on a colored mat-the giver puppet on a red mat and the receiver puppet on a blue mat (see Fig. 3). The child sat on a green mat. Each mat was placed on the corner of a cloth triangle (each side $1 \mathrm{~m}$ ). The moderator sat to the child's right (not on a mat). She informed the child and puppets that the colors of their respective mats would be their assigned colors for the games that they would play next.

An overview of the Sharing tasks procedure is provided in Fig. 4. First, the moderator pulled out a single jingle box (or marble run; order counterbalanced across children), explained how the game is

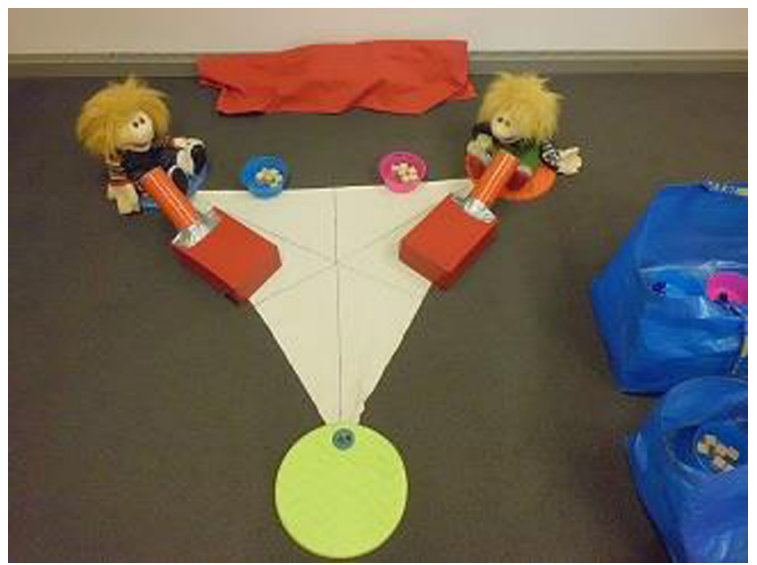

Fig. 3. Setup for the Sharing tasks, showing the cloth triangle, the two puppets (one on a blue mat and one on a red mat), and the green mat for the child. This setup shows the jingle boxes; the setup was identical for the marble runs. (For interpretation of the references to color in this figure legend, the reader is referred to the web version of this article.)

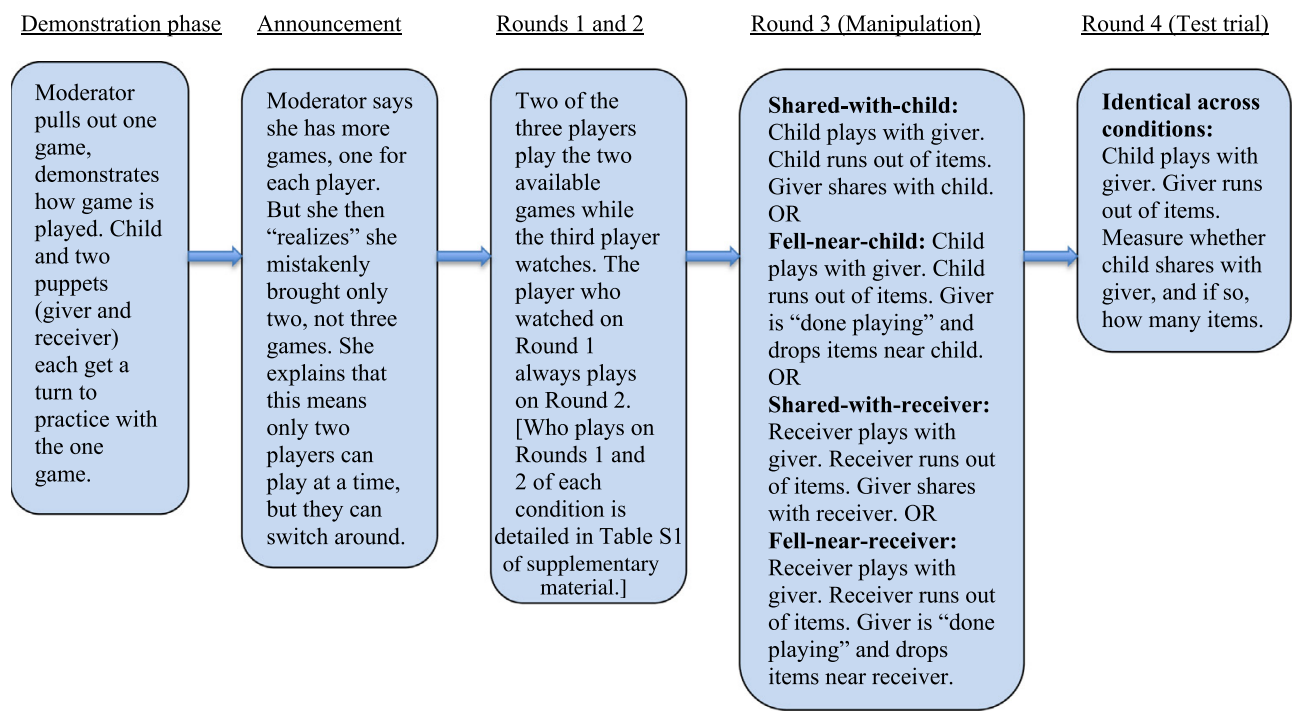

Fig. 4. Flow chart of the Sharing tasks. This was the procedure for one game (e.g., jingle boxes). The procedure was identical for the second game (e.g., marble run). All children then received the three exploratory tasks (not shown in the flow chart). 
played, and played one item (cube or marble for jingle box or marble run, respectively) herself. She then gave each player a turn to practice the game on the one jingle box (marble run). Specifically, she first placed one item in the child's hand and, if necessary, encouraged the child to play, and then she placed another item on the floor near the child and again encouraged the child to play. She then placed one item on the floor near the giver puppet, who played successfully and expressed excitement about playing, and then placed one item on the floor near the receiver puppet, who also played successfully and expressed excitement.

After this demonstration phase, the moderator announced that she had two more jingle boxes (or marble runs) and that all players could play with their own. However, as she reached into her bag to pull out the two additional games, she pretended to realize that she had mistakenly brought only one more with her, that is, that she had brought a total of only two jingle boxes (or marble runs) with her; she explained that this meant that only two of the players could play at a given time but that they would switch around. (This ensured that in the two Receiver conditions, children were not occupied with their own game and so would notice the giver's actions toward the receiver. It also ensured that on sharing test trials, during which the giver and children played, children knew that only they-and not the receiver puppet-could share with the giver.)

The moderator informed the players that the items for the game were already distributed into various containers in her bag and that the containers were of the three colors assigned to the players. She explained that because only two players could play on a given round, she would pull out two containers from her bag for each round and the colors of those containers would determine which two players played on each round. In fact, the containers were pre-organized in the moderator's bag according to condition, as described next (and as detailed in Table S1 of the online supplementary material).

Shared-with-child and Fell-near-child conditions. The moderator pulled out containers in the giver's and receiver's colors (red and blue, respectively) and explained that this meant that the two puppets would play on the first round and the child would watch. The moderator handed each puppet its container and moved the games close to the puppets. When the puppets were done playing with their 6 items, they neutrally said "So..." to signal to the moderator that they were done and returned their containers to the moderator. (Note that the puppets did not say "All done!" or something similar because we did not want to create a competitive atmosphere given that this might have discouraged children from sharing or prompted children to use up all of their items as quickly as possible.)

The second round then began. The moderator pulled out containers in the child's color (green) and in the receiver puppet's color (blue). The moderator moved one game near the child. The child and receiver puppet each played with their items, with the receiver puppet approximately matching its pace to the child's pace. Once they were done, the receiver said "So..." and returned its container to the moderator. Most children also returned their containers (and the rest were requested to do so by the moderator). During this round, the giver neutrally observed the situation and did not react in any way. Note that these first two rounds were not relevant to the experimental manipulation or test but served to ensure that all players had a turn playing the game before the third (experimental manipulation) round. On the third round, the containers were in the child's and giver puppet's colors, and so the moderator moved one game near the giver. Critically, however, they had an unequal number of items; the child's container had 4 items, whereas the giver's container had either 12 items (in the Shared-with-child condition) or 9 items (in the Fell-near-child condition). What happened next varied between these two conditions.

Shared-with-child. The child and giver played at a matched pace such that when the child ran out of his or her 4 items, the giver still had 8 of its 12 items. The giver now shared half of its remaining items with the child. Specifically, if the child reached for an item or asked for it, the giver handed over 4 items to the child immediately, saying, "Here, I'll give you some. Now you can play some more." If the child did not reach or ask for an item, the giver handed 4 items to the child proactively after $5 \mathrm{~s}$ and said the same sentence. The giver always gave 4 items to the child and then continued to play with its remaining 4 items. (Note that the giver began with 12 items in this condition so that when the child ran out of items, the giver still had 8 remaining and could clearly demonstrate generosity by sharing half of its remaining items. The same logic also applied to the Shared-with-receiver condition described later.) 
Fell-near-child. The child and giver played at a matched pace such that when the child ran out of his or her 4 items, the giver still had 5 of its 9 items. However, the giver did not actively share with the child. Instead, if the child reached for or asked for an item, the giver looked over to the child's game, neutrally said "Hmm," and then looked to its own game and played with 1 more item (thus being left with 4 items). Having the giver play with 1 more item after the child ran out of items made it clear that the giver was not responding to the child's need for items. The giver then neutrally said to itself, "I'm done playing now. I don't need these anymore. I'll take them out, I don't need them," and turned its container over in the direction of the child so that the remaining 4 items fell near the child. The giver then said, "But I do need this [the container]," took its container, turned away from the child, and appeared to be busy. If the child did not reach or ask for an item, the giver behaved in the same way after $5 \mathrm{~s}$.

The giver stopped playing and turned away in the Fell-near-child condition because we wanted the child to feel comfortable in taking the giver's 4 items that fell near the child. That is, if the giver had continued to play or to face the games, the child may have thought that the giver would want to use the 4 fallen items as well and, thus, may have been hesitant to take the items. However, if the child still did not take any of the 4 items within $15 \mathrm{~s}$ after the items fell near him or her, the moderator pointed to the items and said to the child, "Look, there are some more [items] lying there." If the child did not take the items in the next $5 \mathrm{~s}$, the moderator said, "I'm sure you can play with those too." If the child still did not take the items, the moderator reiterated, "You can take those and play with them if you like." After waiting another $5 \mathrm{~s}$, the moderator said, "Okay, then let's play another round," and she began the fourth and final round of that game.

In both conditions, the third round ended either when the child had played with all 4 items or $30 \mathrm{~s}$ after the items were given to or fell near the child. During the third round, the receiver puppet simply observed the situation neutrally and did not react in any way, and the puppeteers looked neutrally at their own puppets.

Shared-with-receiver and Fell-near-receiver conditions. The two conditions in which the receiver puppet was the beneficiary were very similar in structure to the Child conditions with the exception of who played in which round. On the first round, the child and giver puppet each played with 6 items. On the second round, the child and receiver puppet each played with 6 items. Again, these first two rounds simply ensured that all players had a turn playing the game before the third round.

On the third (manipulation) round, the giver and receiver puppets played while the child watched. Critically, the containers again had an unequal number of items; the receiver's container had 4 items, whereas the giver's container had either 12 items (Shared-with-receiver) or 9 items (Fell-nearreceiver). What happened next varied between these two conditions.

Shared-with-receiver. The two puppets played at a matched pace such that when the receiver ran out of items, the giver still had 8 items remaining. The giver now clearly demonstrated goodwill by sharing half of its remaining items with the receiver. Specifically, when the receiver ran out of items, it said in a mildly sad tone, "Ohh." The giver responded by handing 4 items to the receiver, saying exactly as in Shared-with-child, "Here, I'll give you some. Now you can play some more." The receiver then said in a mildly happy tone, "Oh, more [items]," and played with all 4 . The giver also played with its remaining 4 items at the same pace.

Fell-near-receiver. The two puppets played at a matched pace such that when the receiver ran out of items, the giver still had 5 items remaining. However, the giver did not actively share with the receiver. Specifically, when the receiver ran out of items, it said in a mildly sad tone, "Ohh." The giver now behaved and spoke exactly as in Fell-near-child, first looking over to the child's game and neutrally saying, "Hmm," and then playing with 1 more item and saying to itself, "I'm done playing now. I don't need these anymore. I'll take them out, I don't need them," and turning its container over in the direction of the receiver so that the remaining 4 items fell near the receiver. The giver then said, "But I do need this [the container]," took its container, turned away from the receiver, and appeared to be busy. The receiver then said in a mildly happy tone, "Oh, more [items]," and played with all 4 . In both conditions, the time between the giver placing the items near the receiver and the receiver playing with all 4 items was approximately $30 \mathrm{~s}$. 
The fourth and final round was the sharing test trial and was identical in all four conditions. The moderator announced that she had only two containers remaining, which meant that this would be the final round of this game. She then pulled out two containers: one with 12 items for the child and one with 4 items for the giver. The giver matched the child's pace of play such that it ran out of items when the child had 8 items remaining. It then provided increasingly more explicit cues of its need in three 5-s phases. First, it simply looked into its own container and said "Ohh" (without looking at the child). If the child did not respond within $5 \mathrm{~s}$, the giver shook its container and said "Ohh" (without looking at the child). If the child did not respond within $5 \mathrm{~s}$, the giver alternated its gaze between the child and its own container twice and said, "I don't have any [items] left. I can't play anymore." If the child still did not respond, the puppet continued to alternate gaze between child and its own container for another $10 \mathrm{~s}$. The trial ended after a total of $30 \mathrm{~s}$ elapsed or the child ran out of items. If at any point the child responded to one of the giver's cues, the giver continued to provide that level of cue as long as the child still had items or until the remaining time had elapsed.

This concluded the first game. The moderator put away the materials of the first game, pulled out the second game, and demonstrated how it was played. The child and two puppets played the four rounds (including the test trial) exactly like the first game and in the same condition. This concluded the Sharing tasks.

\section{Exploratory tasks}

To explore potential reciprocity effects outside the immediate sharing context, we also presented children with three exploratory tasks after the Sharing tasks. The tasks were conducted in a fixed order as follows.

Forced-choice helping. This task was adapted from Vaish et al. (2010). The moderator involved the child in putting away the games. In the meantime, the puppets each brought out from behind them a color-matching game consisting of a box with four holes marked by different colors into which balls of matching colors could be placed. Each puppet also brought out a container holding three balls; both containers were missing a yellow ball (see Fig. 5). The puppets placed their respective games in front of themselves and placed the containers with the balls next to the games. They placed one yellow ball in the child's corner of the cloth triangle, and the child's (green) mat was moved approximately $0.5 \mathrm{~m}$ from the ball. Now the moderator brought the child back to the triangle and asked the child to sit on

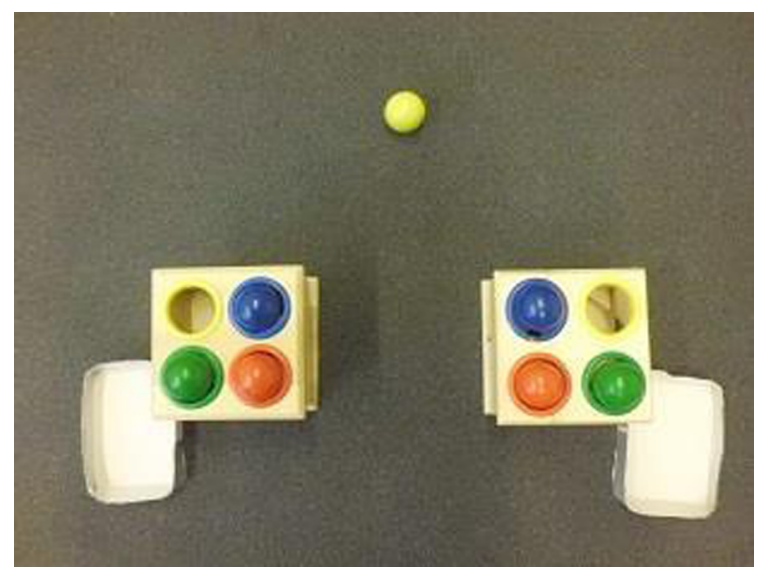

Fig. 5. The color-matching game used in the Forced-choice helping task, with the one yellow ball that both puppets needed placed in the middle. (For interpretation of the references to color in this figure legend, the reader is referred to the web version of this article.) 
his or her green mat, facing the puppets and the yellow ball. The moderator sat behind the child, held the child, and kept time.

The two puppets simultaneously but individually played their respective games. After placing the three available balls in the matching slots, both puppets simultaneously reached for the yellow ball in the middle and maintained this reach, looking only at the ball. If the child did not act within $15 \mathrm{~s}$, the moderator asked the child to "give the ball." If the child still did not react within $15 \mathrm{~s}$, the moderator asked the child to point to the person he or she wanted to give the ball to. As a last resort, the moderator sat between the puppets, held up the ball, and asked the child to give it or point to the person he or she wanted to give it to. Whoever the child chose simply accepted the ball neutrally. If the child did not respond within $1 \mathrm{~min}$, the Forced-choice helping test ended. The puppets moved the colormatching games and containers behind them, out of view of the child.

Preference. The two puppets now sat neutrally on their mats, and the moderator asked the child which puppet he or she would like to play with the next time. If the child did not respond within $10 \mathrm{~s}$, the moderator repeated the question and again waited for $10 \mathrm{~s}$. If the child still did not respond, the moderator moved on to the Distribution task.

Distribution. This final task was adapted from Vaish, Carpenter, and Tomasello (2011). The moderator placed a small container in front of each puppet and gave the child 3 cloth flowers to distribute to the two puppets as the child wanted. If the child did not distribute all the flowers or asked the moderator for guidance, the moderator encouraged the child to make a decision. When the child was done, the moderator asked the child why he or she had given more flowers to the giver or receiver puppet (whichever received more flowers). This concluded the exploratory tasks and the study.

\section{Coding and reliability}

All coding was conducted by coders who were blind to hypotheses. For the Sharing tasks, coders coded the number of items that children shared with the giver puppet during the two sharing test trials. For each trial, the number could range from 0 to 8 . For the Forced-choice helping task, the coders coded whom children chose to give the yellow ball to (giver, receiver, or neither puppet). For the Preference task, they coded whom children pointed to or named. Children's responses on the Preference task could be coded as "giver," "receiver," or "no clear choice" (which included "neither," "both," and unclear or no responses). For the Distribution task, coders coded how many flowers children distributed to the giver and how many of the 3 flowers they distributed in total. Children's justifications for their distribution were also coded. However, because nearly all of the justifications were irrelevant to the main goals of the study (typical responses were "So that she has two," "Because I had three," and "I don't know"), these were not analyzed and are not discussed further.

To assess reliability, an independent coder (blind to conditions and hypotheses) coded a randomly selected $25 \%$ of children $(n=16)$. Reliability was perfect on both sharing test trials and all exploratory tasks (all $\kappa \mathrm{s}=1.00)$.

To account for some low-level explanations and obtain more detailed information about children's behavior, a few additional measures were also coded (and reliability was assessed on a randomly selected $25 \%$ of children). Specifically, on the third round of each game, coders coded (a) the duration of time that children attended to the game situation (puppets, puppeteers, games, game items, or moderator) as a proportion of the total duration of the trial (interrater reliability: intraclass correlation coefficient $[\mathrm{ICC}]=.984, p<.0005$ ); (b) in the two Child conditions, when children ran out of items, whether they spontaneously asked for items from the giver (reliability: $\kappa=1.00$ ); and (c) in the Fell-near-child condition, whether children took the items that fell near them and, if so, whether they did so spontaneously or on being prompted by the moderator $(\kappa=1.00)$. In addition, on the sharing test trials, the coders coded whether children who shared with the giver did so after Prompt 1,2 , or $3(\kappa=.79)$. 


\section{Results}

\section{Sharing test trials}

For the sharing test trials, we investigated the statistical significance of the two predictor variables beneficiary and action on the number of items shared as well as on the number of children who shared.

\section{Number of items shared}

For this analysis, we ran a generalized linear mixed model (GLMM; Bates, Maechler, \& Bolker, 2014) with Poisson error distribution in $R$ ( $R$ Core Team, 2015). We included the Beneficiary $\times A$ ction interaction as a fixed factor and gender, trial ( 1 or 2 ), type of game on Trial 1 (jingle boxes or marble runs), and side on which the giver sat (left or right of child) as control factors. In addition, we included random intercepts both for the participant and for the experimenter who played the giver (Schielzeth \& Forstmeier, 2009). The statistical significance of the Beneficiary $\times$ Action interaction as well as of the other control factors was tested with a likelihood ratio test (drop1 function in R) comparing a full model with reduced models without the respective factors. For pairwise comparisons, we averaged data across the two test trials and computed Wilcoxon exact tests.

As predicted, the Beneficiary $\times$ Action interaction was statistically significant, indicating that the number of items children shared varied significantly as a function of both who had previously received items from the giver (child or receiver) and the giver's intent: GLMM, $\chi^{2}(d f=1)=5.24, p=.02$ (see Fig. 6). Specifically, children shared more items with the giver in the Shared-with-child condition $(M=2.15, S D=1.54)$ than in the Fell-near-child condition $(M=0.33, S D=0.95), U=325.5,95 \%$ confidence interval $(\mathrm{CI})[0.5,3.5], p<.001$. There was no difference in the number of items shared in the Shared-with-receiver condition $(M=1.05, S D=1.57)$ compared with the Fell-near-receiver condition $(M=0.95, S D=1.82), U=219.5,95 \% \mathrm{CI}[-0.5,0.5], p=.53$. Children also shared more items in the Shared-with-child condition compared with both the Shared-with-receiver condition, $U=284,95 \%$ $\mathrm{CI}[0,3], p=.02$, and the Fell-near-receiver condition, $U=285.5,95 \% \mathrm{CI}[0,3], p=.01$. None of the control effects was statistically significant, all $p s>.085$. Thus, children clearly showed the greatest reciprocity toward the benefactor who showed goodwill toward them.

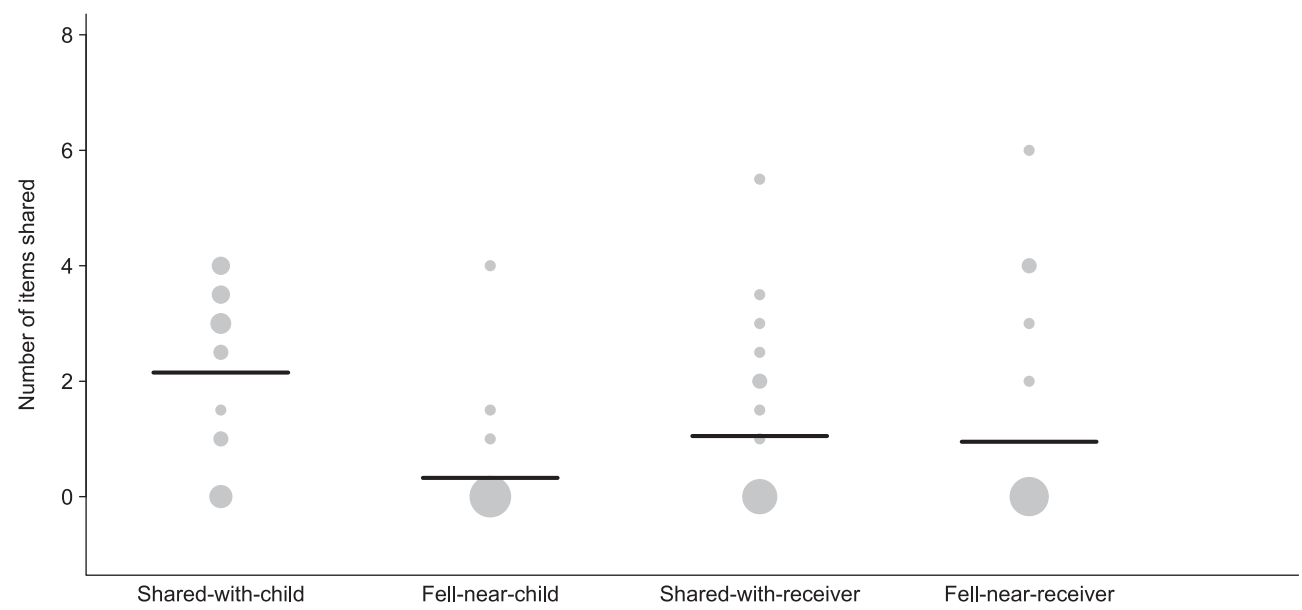

Fig. 6. The number of items children shared during the test trials in each condition. Data are averaged across both test trials. The distributional information is presented through the size of the circles; the smallest circles represent one participant, and the largest circle (in Fell-near-child) represents 17 participants (for detailed information about the numbers of children represented in this figure, see Table S2 in supplementary material). Horizontal black lines represent group means. 
Number of children who shared

For this analysis, we pooled the two test trials, assigning children a value of 1 if they shared at all on at least one test trial and a value of 0 if they did not share on either test trial. The results were very similar for each trial separately (see Table 1 ). We conducted a logistic regression (generalized linear model [GLM] with a binomial error structure), with the Beneficiary $\times$ Action interaction as a fixed factor and gender, type of game on Trial 1, which experimenter played the giver, and side on which the giver sat as control factors. Statistical significance was again tested with the drop1 function in R comparing a full model with reduced models without the respective factors.

As with the number of items shared, the analysis of the number of children who shared on at least one test trial also revealed a Beneficiary $\times$ Action interaction: GLM, $\chi^{2}(d f=1)=4.27, p=.039$. In support of our hypothesis, children were most likely to share in the Shared-with-child condition than in any other condition. Pairwise comparisons using Fisher's exact tests revealed that children were more likely to share with the giver in the Shared-with-child condition (15 of 20 children) than in the Fellnear-child condition ( 3 of 20 children), 95\% CI [.01, .39], $p=.0008$, whereas children's likelihood of sharing did not differ significantly between the Shared-with-receiver condition ( 8 of 20 children) and the Fell-near-receiver condition (5 of 20 children), 95\% CI [0.1, 3.1]), $p=.72$. The model did not reveal any other significant effects, all ps $>.45$.

\section{Prompt at which children shared}

We aimed to analyze the prompt $(1,2$, or 3$)$ after which children first shared during each sharing trial. Children who did not share were not included in these analyses. This resulted in very small sample sizes for all conditions except Shared-with-child because very few children in the other three conditions shared at all (see Table 1). Dividing these small numbers further into those who shared after Prompts 1, 2, and 3 resulted in extremely small numbers per cell (just 1 or 2 children in most cells). This rendered it difficult to conduct meaningful statistical analyses on this measure. Thus, we did not analyze this measure.

\section{Additional analyses}

We conducted additional analyses in order to address possible low-level differences between conditions and provide additional information about children's behavior. We describe each briefly here; further descriptive information and details of analyses are provided in the supplementary material.

Attention differences across conditions. Because children were themselves playing during the third (manipulation) rounds of the games in the two Child conditions but not in the two Receiver conditions, it is possible that children paid more attention, and thus were simply more likely to notice important aspects of the game situation (such as whether or not the giver intended to share), in the Child conditions than in the Receiver conditions. However, analyses of the proportion of time that children attended to the game situation during the third round of each game revealed that children were highly attentive to the game situations in all conditions (all mean proportions $>.95$ ) and that there were no differences in attentiveness between the Child and Receiver conditions.

Requesting items in child conditions. In the Child conditions, no child ever explicitly asked the giver for items, but several children behaved in ways that could be considered subtle or implicit requests (such

Table 1

Numbers of children who shared with the giver puppet.

\begin{tabular}{llll}
\hline Condition & Test Trial 1 & Test Trial 2 & Both trials pooled $^{\text {a }}$ \\
\hline Shared-with-child & 13 & 14 & 15 \\
Fell-near-child & 2 & 2 & 3 \\
Shared-with-receiver & 6 & 8 & 8 \\
Fell-near-receiver & 4 & 5 & 5 \\
\hline
\end{tabular}

Note. The total number of children in each cell was 20.

a The "Both trials pooled" column represents numbers of children who shared on at least one test trial. 
as stating that they did not have any more items or placing their container near the giver). However, these children did not later share more or less with the giver than children who did not show these behaviors, all $p s>.242$.

Taking items in Fell-near-child condition. Five of the 20 children in Fell-near-child condition did not take the giver's items ( 2 did not take them during either game, and 3 took them during the second game only). These 5 children were as likely to subsequently share with the giver as the 15 children who took items on both trials (Fisher's exact test, $p=1.00$ ). In addition, children who took the items spontaneously versus after being prompted by the moderator also did not differ in their subsequent sharing, $p s>.565$.

\section{Exploratory tasks}

\section{Forced-choice helping}

Two children did not hand the yellow ball to either puppet and, thus, were excluded from this analysis. We conducted a logistic regression with a binomial error structure, with the Beneficiary $\times$ Action interaction as a fixed factor; gender, type of game on Trial 1, which experimenter played the giver, and side on which the giver sat as control factors; and the puppet that children handed the yellow ball to (giver or receiver) as the binary dependent measure. Neither the interaction term nor any of the main effects were found to contribute significantly to the model, all $p s>.29$. Thus, in this first task outside the immediate context within which children received benefits, there was no evidence that children selectively helped either puppet more.

\section{Preference}

Of the 80 children, 24 were coded as having made "no clear choice" and, thus, were excluded from the analysis ( $n=6$ in Shared-with-child, $n=3$ in Fell-near-child, $n=8$ in Shared-with-receiver, and $n=$ 7 in Fell-near-receiver). We conducted a logistic regression with a binomial error structure, with the Beneficiary $\times$ Action interaction as a fixed factor; gender, type of game on Trial 1 , which experimenter played the giver, and side on which the giver sat as control factors; and the puppet for whom children expressed a preference (giver or receiver) as the binary dependent measure. Again, neither the interaction term nor any of the main effects were found to contribute significantly to the model, all $p s>.48$.

\section{Distribution}

All children distributed all three flowers between the two puppets. Because the data from this task fulfilled the assumptions of an analysis of variance (ANOVA), a between-participants ANOVA was conducted, with the Beneficiary $\times$ Action interaction, gender, type of game on Trial 1, which experimenter played the giver, and side on which the giver sat as the independent variables and number of flowers distributed to the giver puppet as the dependent variable. This did not reveal a significant Beneficiary $\times$ Action interaction $(p=.127)$, but it did reveal a marginal main effect of beneficiary, $F(1,71)=3.44, p$ $=.068$, such that children distributed more flowers to the giver in the two Child conditions $(M=1.58$, $S D=0.75)$ than in the two Receiver conditions $(M=1.28, S D=0.75)$. The analysis also revealed a marginal main effect of action, $F(1,71)=3.44, p=.068$, such that children distributed more flowers to the giver in the Shared conditions $(M=1.58, S D=0.81)$ than in the Fell conditions $(M=1.28, S D=0.68)$. Thus, children's distribution of the flowers seemed to weakly take into account who had received benefits and whether the benefits were intended, mirroring the pattern seen in children's sharing on the sharing test trials. However, these factors did not interact to elicit the specificity in children's prosociality that was evident on the Sharing task.

The analysis also revealed an unexpected significant main effect of which experimenter played the giver, $F(2,71)=3.16, p=.049$. Children distributed more flowers to the giver when the giver was played by the puppeteer who was involved in testing all children $(M=1.63, S D=0.81)$ than when the giver was played by the other two puppeteers $(M=1.25, S D=0.67$ for the experimenter who tested the first 64 children; $M=1.13, S D=0.64$ for the experimenter who tested the remaining 16 children). Note, however, that this effect of experimenter was found on this final task, and even here it did not interact with any of the other factors. 


\section{Discussion}

We aimed to identify the mechanisms underlying young children's reciprocity. Specifically, children might reciprocate whenever they receive benefits (regardless of the benefactor's intentions) or might show prosociality toward all generous individuals (regardless of whom those individuals benefited). A richer possibility is that children show the greatest reciprocity when these two factors interact, that is, toward a benefactor who intentionally benefits them and, thus, demonstrates goodwill toward them.

Our results clearly supported this last possibility: Children who received benefits through the benefactor's good intentions subsequently shared significantly more with the benefactor than children who received benefits without the benefactor intending to benefit them and than children who observed the benefactor benefiting someone else. Thus, children's reciprocity is not only a response to receiving benefits, nor is it driven only by a mechanism of rewarding or preferring benevolent individuals; rather, it takes both of these factors into account such that children are especially motivated to benefit those benefactors who have demonstrated goodwill toward them. This specificity in children's reciprocity is likely an important mechanism by which early cooperative relationships are formed and maintained (Nowak, 2006; Trivers, 1971).

What psychological mechanism might underlie this specificity? Our design allows us to rule out two "thinner" mechanisms: a positive mood that results simply from receiving benefits and a general desire to affiliate with or reward benevolent individuals. Instead, our findings are consistent with the possibility of gratitude (Bonnie \& de Waal, 2004; Trivers, 1971). Gratitude is a positive emotion that is experienced when one receives a benefit through the good intentions of another, and it urges the grateful person to respond prosocially in turn (e.g., Algoe, 2012; Bartlett, Condon, Cruz, Baumann, \& DeSteno, 2012; DeSteno, Bartlett, Baumann, Williams, \& Dickens, 2010; McCullough, Kimeldorf, \& Cohen, 2008; see Ma, Tunney, \& Ferguson, 2017, for a review and meta-analysis). Importantly, gratitude urges more prosociality than simply a positive mood or positive evaluation of benefactors. For instance, adults reported feeling more gratitude and displayed greater reciprocity if they received a benefit from another person than by chance (Tsang, 2006b; Tsang, Schulwitz, \& Carlisle, 2012; see also Bartlett \& DeSteno, 2006) and if a benefactor helped them out of benevolent motives rather than self-serving motives (Tsang, 2006a). Our results follow this pattern precisely, hinting at the possibility that the psychological mechanism underlying young children's reciprocity might be gratitude.

Of course, we did not directly measure children's emotional responses. Our proposal of gratitude as the underlying mechanism, thus, is necessarily tentative, and other mechanisms are certainly possible as well. For instance, perhaps children were motivated by indebtedness, that is, the discomfort or feeling of obligation to repay someone who provided assistance (Greenberg, 1980; Watkins, Scheer, Ovnicek, \& Kolts, 2006). Our study cannot rule out this possibility. However, work with adults suggests that gratitude is distinct from indebtedness in important ways. For one, whereas gratitude is sensitive to a benefactor's intentions, indebtedness seems not to be (Peng, Nelissen, \& Zeelenberg, 2017; Tsang, 2006a). Gratitude also motivates greater prosocial behavior than indebtedness alone (Tsang, 2006a; Watkins et al., 2006). In addition, whereas gratitude motivates upstream reciprocity (i.e., providing benefits to people other than one's benefactor), indebtedness might not do so to the same extent (Bartlett \& DeSteno, 2006; Chang, Lin, \& Chen, 2012; Fredrickson, 2012; Halali, Kogut, \& Ritov, 2017; Nowak \& Roch, 2007; Peng et al., 2017). Two recent developmental studies show that preschool and school-age children do engage in upstream reciprocity (Claidière et al., 2015; Leimgruber et al., 2014), hinting that children's reciprocity is not motivated (only) by indebtedness. Importantly, however, they did not provide evidence for gratitude because neither study varied the benefactor's intentions or whom the benefactor benefited, both of which are important factors for gratitude (as noted above). Thus, children in those studies may have been motivated by a different mechanism such as imitating the benefactor's behavior. Future work on upstream reciprocity could vary these two important factors (as in the current study) to more systematically test for gratitude. 
Two further alternative mechanisms are worth mentioning. One is punishment, that is, that children may have been punishing the nonsharing puppet in the Fell conditions by withholding resources from that puppet (e.g., Salali, Juda, \& Henrich, 2015; Vaish et al., 2010). Note, however, that children also showed reduced sharing in the Shared-with-receiver condition, suggesting that children's behavior was likely not driven (only) by punishment. Finally, children's sharing might be explained by reputation management such as the desire to appear generous in front of the generous puppet (e.g., Engelmann, Herrmann, \& Tomasello, 2012). Children did not, however, show the same increased generosity in the Shared-with-receiver condition, hinting that they were at least not managing their reputation in that condition. Nonetheless, distinguishing among these various possible mechanisms underlying children's reciprocity is a fascinating direction for future research.

It is important to delve deeper into our result that children did not share significantly more resources with an individual who intended to share with a third party (Shared-with-receiver condition) than with an individual who did not (Fell-near-receiver condition). First, it is worth remembering that the levels of sharing in all but the Shared-with-child condition were rather low. Thus, there may have been something of a floor effect across those three conditions, making it difficult to detect any differences between them. It is possible that a larger sample size or a more sensitive measure (e.g., a measure of children's affect rather than their sharing) would reveal that children do respond more positively to an intentional third-party benefactor than to an unintentional one (although perhaps still not as much as to an intentional first-party benefactor). This will be an important step for future work.

At the same time, it can be instructive to consider the implications of our results if they were taken at face value. In particular, the absence of a statistical difference between children's prosocial behavior in Shared-with-receiver versus Fell-near-receiver seems to contradict recent findings that toddlers and even infants prefer and reward individuals who have positive intentions toward others (e.g., Dahl et al., 2013; Hamlin et al., 2011). Importantly, however, children in those studies showed a preference for individuals with positive intentions over those with negative intentions and so might have primarily been avoiding the individual with negative intentions. In contrast, children in our study were faced with positive or neutral intentions (the giver's intentions in the Fell conditions were not negative but simply unrelated to the other's need) and, thus, needed to show preference for the person with positive intentions. It is possible that children prefer and reward those with positive intentions when they are themselves the recipients of those intentions but not when a third party is the recipient.

Prior work lends support to this proposal. In a study where children were the recipients, they distinguished and preferentially helped someone who had exhibited positive intentions rather than neutral intentions toward them (Dunfield \& Kuhlmeier, 2010), whereas in a study where children observed third-party interactions, they did not preferentially help a person with positive intentions rather than neutral intentions, although they were less prosocial toward a person with negative intentions than with neutral intentions (Vaish et al., 2010). Thus, young children track others' intentions and direct less help toward individuals with negative intentions even in third-party interactions, but they might appreciate and reciprocate toward individuals with positive intentions only in firstparty interactions. This is consistent with our findings that children's reciprocity differed between the two Child conditions but not between the two Receiver conditions and that children showed greater reciprocity in the Shared-with-child condition than in the Shared-with-receiver condition. In sum, it will be important in future work to examine whether young children perhaps are sensitive to third-party benefactors' positive intentions and our failure to find this sensitivity was due to our sample size or methodology; if they are not, then it will be fascinating to examine when during development this sensitivity emerges.

We conducted three exploratory tasks in order to gather some information on children's prosocial behavior and social decisions outside the immediate context within which they received benefits. However, these tasks did not reveal the same specificity as the Sharing task; children did not show greater prosocial behavior toward or preference for the benefactor who had shown goodwill toward them. This hints that once children have been able to reciprocate right away, they might not continue to bestow favors on the benefactor, at least not until the benefactor benefits them again. Alternatively, perhaps children primarily reciprocate by providing benefits that are similar to the benefits they 
received (i.e., game items in the current study) but not different benefits. Because our exploratory tasks followed the primary Sharing task and also involved different benefits than those that children received, we cannot determine which explanation is correct or whether both explanations played a role. In any case, our pattern of results seems consistent with the nature of direct reciprocity, which is typically conceived of as taking turns giving and taking resources of similar value so that both individuals benefit in the long term (e.g., Axelrod \& Hamilton, 1981; Fehr \& Fischbacher, 2004; Trivers, 1971).

The lack of effects in our exploratory tasks is also consistent with some other recent work on social processes. For instance, Vaish, Carpenter, and Tomasello (2016) found that after 3-year-old children had caused someone harm, they showed reparative behavior immediately after the mishap but not during later phases of the procedure and not in novel contexts. Similarly, Michaelson and Munakata (2016) found that 3- to 5-year-olds were more willing to delay gratification for a reward promised by a trustworthy experimenter than by a non-trustworthy experimenter, but this differentiation did not carry over into a subsequent preference task. Together, these findings suggest that young children's social evaluations and responses affect their immediate behavior but might not carry over into later or novel situations. The nature and scope of this phenomenon will be interesting to consider in future work.

Some limitations of our study should be acknowledged. First, children interacted with puppets rather than with real people. We made this choice (following Warneken \& Tomasello, 2013) because puppets offer several advantages over peers or adults. Having peers as interaction partners proves to be impractical for studies like the current one because it is challenging to train young children to behave in a highly scripted manner, especially given the complex nature of our method, the precise timing of each prompt or action, and the importance of carefully controlled emotional or neutral expressions at prespecified times. Adults as interaction partners can also prove to be problematic because some work suggests that children are less forthcoming in their interactions with adults than with puppets. For instance, in the context of moral transgressions, children protest and tattle against puppet transgressors but seem more reluctant to speak up against adult transgressors, perhaps because adults are seen as authority figures (Kenward \& Östh, 2012). Although the current study examined situations of reciprocity rather than transgressions, it was nonetheless important to ensure that children were comfortable in responding to their interaction partners. A final possibility would have been to use videos of human actors. However, although videos provide excellent experimental control, they do not lend themselves to live ongoing interactions, and our aim in the current study was specifically to examine children's contingent reciprocity with an interaction partner. Thus, puppets were a good solution for the specific needs of the current study. Nonetheless, puppets may add an additional level of complexity or distract children from the task, raising issues of ecological validity. Thus, it will be important in future work to use alternative approaches in order to establish the robustness and generalizability of the current findings.

We also focused on one age group ( 3 years) because prior work robustly indicates that children at this age share reciprocally and our aim was to tease apart the mechanisms underlying such reciprocal sharing. Nonetheless, future work might successfully elicit reciprocal sharing in younger ages using a simpler paradigm and might then be able to assess whether reciprocity at those younger ages shows the same specificity that we have demonstrated in 3-year-olds. In sum, more work is needed to shed light on the development and bounds of the effect shown here.

Finally, we consider the differences between our Shared and Fell conditions. Although our aim in designing these conditions was to manipulate the giver's intentions, the giver's behavior also varied in other ways between these conditions. For instance, in the Shared conditions the giver continued playing the game and so presumably valued the items that it shared, whereas in the Fell conditions the giver did not continue playing and could have been perceived as no longer valuing the items. Thus, children may have responded to the difference in the perceived value of the given items rather than, or in addition to, the difference in the giver's intentions. This would not undermine our findings because giving something valuable to someone is another important way of indicating one's goodwill toward that person (McCullough, Kilpatrick, Emmons, \& Larson, 2001; Tesser, Gatewood, \& Driver, 1968). 
Nonetheless, it will be interesting in future work to tease apart intentions from value of resources in order to more precisely spell out the mechanisms underlying early reciprocity.

A second (related) difference is that in the Fell conditions the giver purposefully dropped its items and then turned away and seemed to disengage from the game that the child presumably liked. ${ }^{3}$ It is possible that children were put off by this disengagement and, thus, shared less with the giver in the Fell conditions. However, although the child and puppets played the same games, they always played independently (on separate apparatuses) and so the giver puppet's disengagement did not directly affect the child's ability to continue playing. Still, perhaps children perceived the game playing as a cooperative activity rather than an individual activity. But note that in prior work on the development of cooperation, when an experimenter temporarily disengaged from a cooperative activity that he had been engaging in with children, children as young as 18-24 months attempted to reengage the experimenter such as by placing the game closer to him (Warneken, Chen, \& Tomasello, 2006). This is in contrast to our finding that children were less likely to share resources with the puppet who had previously disengaged from the game. Thus, it seems unlikely that children shared less in the Fell conditions because they were put off by the giver puppet's disengagement from the game. Still, we acknowledge that the puppet's behavior in the Fell versus Shared conditions was different in potentially important ways, and if possible, future work should attempt to further reduce such differences in order to minimize alternative interpretations.

To conclude, our findings demonstrate that 3-year-old children's direct reciprocity is particularly aimed at those benefactors who have demonstrated goodwill toward them. Thus, young children skillfully identify those individuals who mean them well and, in turn, invest more in the well-being of those individuals. This specificity in early reciprocity is likely an important mechanism by which cooperative relationships are formed and maintained.

\section{Acknowledgment}

This research was supported by a Dilthey Fellowship awarded to Amrisha Vaish by the Volkswagen and Fritz Thyssen Foundations.

\section{Appendix A. Supplementary material}

Supplementary data associated with this article can be found, in the online version, at https://doi. org/10.1016/j.jecp.2017.11.005.

\section{References}

Aknin, L. B., Hamlin, J. K., \& Dunn, E. W. (2012). Giving leads to happiness in young children. PLoS ONE, 7(6), e39211.

Algoe, S. B. (2012). Find, remind, and bind: The functions of gratitude in everyday relationships. Social and Personality Psychology Compass, 6, 455-469.

Axelrod, R., \& Hamilton, W. D. (1981). The evolution of cooperation. Science, 211, 1390-1396.

Bartlett, M. Y., Condon, P., Cruz, J., Baumann, J., \& DeSteno, D. (2012). Gratitude: Prompting behaviours that build relationships. Cognition and Emotion, 26, 2-13.

Bartlett, M. Y., \& DeSteno, D. (2006). Gratitude and prosocial behavior: Helping when it costs you. Psychological Science, 17, 319-325.

Bates, D., Maechler, M., \& Bolker, B. M. (2014). Ime4: Linear mixed-effects models using Eigen and S4 (R package version 1.1-7). http://CRAN.R-project.org/package=lme4\%3E.

Bonnie, K. E., \& de Waal, F. B. M. (2004). Primate social reciprocity and the origin of gratitude. In R. A. Emmons \& M. E. McCullough (Eds.), The psychology of gratitude (pp. 213-229). New York: Oxford University Press.

Carlson, M., Charlin, V., \& Miller, N. (1988). Positive mood and helping behavior: A test of six hypotheses. Interpersonal Relations and Group Processes, 55, 211-229.

Chang, Y.-P., Lin, Y.-C., \& Chen, L. H. (2012). Pay it forward: Gratitude in social networks. Journal of Happiness Studies, 13, 761-781.

\footnotetext{
${ }^{3}$ We designed the Fell conditions in this way because pilot work indicated that if the giver's items "accidentally" fell out of the container or the giver continued facing the games and the other players, children were hesitant to pick up the items and play with them, presumably because they were unsure of whether the giver might still want to use the items. Because it was important to our research question that children pick up the giver's items and play with them, we modified the paradigm such that the giver purposefully dropped the items, stated that it was done playing and did not need the items anymore, and turned away.
} 
Claidière, N., Whiten, A., Mareno, M. C., Messer, E. J. E., Brosnan, S. F., Hopper, L. M., ... McGuigan, N. (2015). Selective and contagious prosocial resource donation in capuchin monkeys, chimpanzees, and humans. Scientific Reports, 5. https://doi. org/10.1038/srep07631.

Dahl, A., Schuck, R. K., \& Campos, J. J. (2013). Do young toddlers act on their social preferences? Developmental Psychology, 49, 1964-1970.

DeSteno, D., Bartlett, M. Y., Baumann, J., Williams, L. A., \& Dickens, L. (2010). Gratitude as moral sentiment: Emotion-guided cooperation in economic exchange. Emotion, 10, 289-293.

Dunfield, K. A., \& Kuhlmeier, V. A. (2010). Intention-mediated selective helping in infancy. Psychological Science, 21, $523-527$.

Engelmann, J. M., Herrmann, E., \& Tomasello, M. (2012). Five-year-olds, but not chimpanzees, attempt to manage their reputations. PLOS ONE, 7(10), e48433.

Fehr, E., \& Fischbacher, U. (2004). Social norms and human cooperation. Trends in Cognitive Sciences, 8, 185-190.

Fredrickson, B. L. (2012). Gratitude, like other positive emotions, broadens and builds. In R. A. Emmons \& M. E. McCullough (Eds.), The psychology of gratitude (pp. 145-166). New York: Oxford University Press.

Greenberg, M. S. (1980). A theory of indebtedness. In K. J. Gergen, M. S. Greenberg, \& R. H. Willis (Eds.), Social exchange: Advances in theory and research (pp. 3-26). New York: Plenum.

Halali, E., Kogut, T., \& Ritov, I. (2017). Reciprocating (more) specifically to you: The role of benefactor's identifiability on direct and upstream reciprocity. Behavioral Decision Making, 30, 473-483.

Hamlin, J. K., Wynn, K., Bloom, P., \& Mahajan, N. (2011). How infants and toddlers react to antisocial others. Proceedings of the National Academy of Sciences of the United States of America, 108, 19931-19936.

Kenward, B., \& Dahl, M. (2011). Preschoolers distribute scarce resources according to the moral valence of recipients' previous actions. Developmental Psychology, 47, 1054-1064.

Kenward, B., \& Östh, T. (2012). Enactment of third-party punishment by 4-year-olds. Frontiers in Psychology, 3, 373. https://doi. org/10.3389/fpsyg.2012.00373.

Killen, M., Mulvey, K. L., Richardson, C., Jampol, N., \& Woodward, A. (2011). The accidental transgressor: Morally-relevant theory of mind. Cognition, 119, 197-215.

Leimgruber, K. L., Ward, A. F., Widness, J., Norton, M. I., Olson, K. R., Gray, K., \& Santos, L. R. (2014). Give what you get: Capuchin monkeys (Cebus apella) and 4-year-old children pay forward positive and negative outcomes to conspecifics. PLoS ONE, 9(1), e87035.

Levitt, M. J., Weber, R. A., Clark, M. C., \& McDonnell, P. (1985). Reciprocity of exchange in toddler sharing behavior. Developmental Psychology, 21, 122-123.

Ma, L. K., Tunney, R. J., \& Ferguson, E. (2017). Does gratitude enhance prosociality? A meta-analytic review. Psychological Bulletin, 143, 601-635.

McCullough, M. E., Kilpatrick, S. D., Emmons, R. A., \& Larson, D. B. (2001). Is gratitude a moral affect? Psychological Bulletin, 127, 249-266.

McCullough, M. E., Kimeldorf, M. B., \& Cohen, A. D. (2008). An adaptation for altruism? The social causes, social effects, and social evolution of gratitude. Current Directions in Psychological Science, 17, 281-285.

Michaelson, L. E., \& Munakata, Y. (2016). Trust matters: Seeing how an adult treats another person influences preschoolers' willingness to delay gratification. Developmental Science, 19, 1011-1019.

Nobes, G., Panagiotaki, G., \& Pawson, C. (2009). The influence of negligence, intention, and outcome on children's moral judgments. Journal of Experimental Child Psychology, 104, 382-397.

Nowak, M. A. (2006). Five rules for the evolution of cooperation. Science, 314, 1560-1563.

Nowak, M. A., \& Roch, S. (2007). Upstream reciprocity and the evolution of gratitude. Proceedings of the Royal Society B: Biological Sciences, 274, 605-610.

Olson, K. R., \& Spelke, E. S. (2008). Foundations of cooperation in young children. Cognition, 108, 222-231.

Paulus, M. (2016). It's payback time: Preschoolers selectively request resources from someone they had benefitted. Developmental Psychology, 52, 1299-1306.

Peng, C., Nelissen, R. M. A., \& Zeelenberg, M. (2017). Reconsidering the roles of gratitude and indebtedness in social exchange. Cognition and Emotion. Advance online publication. https://doi.org/10.1080/02699931.2017.1353484.

R Core Team (2015). R: A language and environment for statistical computing. Vienna, Austria: R Foundation for Statistial Computing. http://www.R-project.org.

Salali, G. D., Juda, M., \& Henrich, J. (2015). Transmission and development of costly punishment in children. Evolution and Human Behavior, 36, 86-94.

Schielzeth, H., \& Forstmeier, W. (2009). Conclusions beyond support: Overconfident estimates in mixed models. Behavioral Ecology, 20, 416-420.

Sebastián-Enesco, C., \& Warneken, F. (2015). The shadow of the future: 5-year-olds, but not 3-year-olds, adjust their sharing in anticipation of reciprocation. Journal of Experimental Child Psychology, 129, 40-54.

Tesser, A., Gatewood, R., \& Driver, M. (1968). Some determinants of gratitude. Journal of Personality and Social Psychology, 9, 233-236.

Tomasello, M. (2009). Why we cooperate. Cambridge, MA: MIT Press.

Trivers, R. (1971). The evolution of reciprocal altruism. Quarterly Review of Biology, 46, 35-57.

Tsang, J.-A. (2006a). The effects of helper intention on gratitude and indebtedness. Motivation and Emotion, 30, $198-204$.

Tsang, J.-A. (2006b). Gratitude and prosocial behaviour: An experimental test of gratitude. Cognition and Emotion, 20, 138-148.

Tsang, J.-A., Schulwitz, A., \& Carlisle, R. D. (2012). An experimental test of the relationship between religion and gratitude. Psychology of Religion and Spirituality, 4, 40-55.

Vaish, A., Carpenter, M., \& Tomasello, M. (2010). Young children selectively avoid helping people with harmful intentions. Child Development, 81, 1661-1669.

Vaish, A., Carpenter, M., \& Tomasello, M. (2011). Young children's responses to guilt displays. Developmental Psychology, 47, $1248-1262$.

Vaish, A., Carpenter, M., \& Tomasello, M. (2016). The early emergence of guilt-motivated prosocial behavior. Child Development, $87,1772-1782$. 
Warneken, F., Chen, F., \& Tomasello, M. (2006). Cooperative activities in young children and chimpanzees. Child Development, 77, 640-663.

Warneken, F., \& Tomasello, M. (2013). The emergence of contingent reciprocity in young children. Journal of Experimental Child Psychology, 116, 338-350.

Watkins, P. C., Scheer, J., Ovnicek, M., \& Kolts, R. (2006). The debt of gratitude: Dissociating gratitude and indebtedness. Cognition and Emotion, 20, 217-241. 